\title{
Feasibility study and optimum loading pattern of a multi-ring inflatable intravaginal applicator
}

\author{
Hsiang-Chi Kuo, PhD'., Keyur J. Mehta, MD', Ravindra Yaparpalvi, MS', Linda Hong, PhD', Dinesh Mynampati, MS' \\ Wolfgang A. Tomé, PhD', Shalom Kalnicki, MD' \\ IDepartment of Radiation Oncology, Montefiore Medical Center, Bronx, New York, USA, 2Einstein Institute of Oncophysics, Albert Einstein \\ College of Medicine, Bronx, New York, USA
}

\begin{abstract}
Purpose: A cylinder applicator is the standard treatment device for intravaginal brachytherapy. However, they are limited in their ability to simultaneously spare the organs at risk (OAR), and reduce the hot spot in the vaginal mucosa, while achieving adequate dose conformality. This study aims to compare the dosimetric characteristics of single and multi-channel cylinders, and utilizes volume point dose optimizations to investigate the feasibility and optimum loading method for a multi-ring inflatable intravaginal applicator.

Material and methods: Studies were designed to: (1) test the feasibility of multi-ring applicators, (2) compare dose distributions between different multi-channel applicators and loading patterns, (3) test non-uniform prescription depths around the multi-ring cylinder.

Results: Compared to a cylinder with a single central channel, a cylinder with 6 lumina arranged around the periphery, providing the lumina had adequate distance to the cylinder surface, could reduce dose beyond the prescription depth. However, when the number of outer lumina increased from 6 to 12, no further dose reduction could be achieved and the high dose volume close to the surface of the cylinder increased. Moreover, an additional ring, with lumina further away from the surface, provided increased dose shaping capabilities, allowing for individualized dose distributions.

Conclusions: Dose could be reduced to normal tissue and the inner mucosa, and better conformity was seen to unique anatomical shapes. A modified peripheral loading pattern provided the optimum dose distribution, yielding good conformity, dose sparing at adjacent organs, and dose reduction in the high dose region of the vaginal mucosa.
\end{abstract}

J Contemp Brachytherapy 2013; 5, 2: 93-100

DOI: $10.5114 / \mathrm{jcb} .2013 .35580$

Key words: brachytherapy, endometrial cancer, intravaginal, multi-channel, multi-ring applicator.

\section{Purpose}

Endometrial cancer is the most common female genital tract malignancy in the United States. It is the sixth most common cancer in women worldwide. According to the LOBOCAN project under the World Health Organization (WHO), around 288000 cases of endometrial cancer were recorded and caused nearly 74000 deaths in 2008. The National Cancer Institute (NCI) estimated the number of new cases and deaths from endometrial (uterine corpus) cancer in the United States in 2012 were 47130 and 8 010, respectively. The standard management for endometrial cancer is a total abdominal hysterectomy with bilateral salpingooophorectomy with or without pelvic lymph node sampling [1]. External beam radiation therapy (EBRT) and/or brachytherapy (BT) are integral components in the postoperative adjuvant therapy of selected patients, and in the management of inoperable or recurrent endometrial cancer. In the treatment of recurrent endometrial cancer with EBRT and/or BT, Lin et al. [2] reported 5 and 10 year disease free survival rate
(DFS) of $68 \%$ and $55 \%$, respectively. Jhingran et al. [3] reported 2 and 5 year local control of $85 \%$ and $75 \%$, respectively, and concluded that combination treatment was associated with better control. In postoperative BT, the target is the mucosa of the vaginal cuff, and in some cases, the whole length of the vaginal wall. Since $90 \%$ of vaginal recurrences occur at the vaginal cuff, and only about $10 \%$ in the distal vagina, to avoid vaginal morbidity (vaginal dryness, shortening, stenosis), only the vaginal cuff, the adjacent mucosa, and the upper third of the vagina are treated as the target for brachytherapy [4]. In the American Brachytherapy Society's (ABS) 2005 report [5], 40.7\% and 54.5\% of practices in US treat the upper one-third and upper one-half of the vagina, respectively. Applicator selection in brachytherapy is important. Using a single applicator it can be difficult to treat all anatomical variations and disease presentations. In the same report by the ABS, $90.6 \%$ of practices in the US use single-channel vaginal cylinders in vaginal brachytherapy. $92.3 \%$ and $96.5 \%$ are prescribed at $5 \mathrm{~mm}$ 
depth with and without external beam, respectively. However, cylinders give high doses to the bowel, bladder and rectum.

Another drawback of cylinders is the air gaps that result during procedures since the vagina contains folds and wrinkles. Cameron et al. [6] found 32\% of patients treated with cylinders had air gaps that raised the surface of the vagina more than $2 \mathrm{~mm}$ beyond the surface of the cylinder. It was estimated that this could result in a $13.3 \%$ dose reduction to the vaginal mucosa beyond the air gaps. Richardson et al. [7] also reported $60 \%$ of intra-vaginal brachytherapy procedures have air gaps that can cause a 27\% dose reduction to vaginal mucosa. Miller et al. [8] and Sowards et al. [9] have used Mammosite ${ }^{\circledR}$ (Hologic Inc., Bedford, MA, USA) and Contura ${ }^{\circledR}$ (SenoRx, Inc., Aliso Viejo, CA, USA) balloons in vaginal cuff HDR brachytherapy to reduce air gaps and found that balloons provide excellent coverage to the vaginal cuff.

Multi-channel cylinders have been designed to improve the drawback of single channel cylinders [10-12]. They have the advantage of dose reduction to the bladder and rectum at the expense of increased dose to the surface of the cylinder. In addition to the anisotropic nature of the brachytherapy source, the geometric parameters of the cylinder applicator, which include applicator size, treatment depth, space between source tip and surface of cylinder apex, and the curved portion of the cylinder dome, can affect and limit the optimum dose distribution, especially when it is optimized with fixed points or fixed distance to the dwell positions $[13,14]$. In our study, we aim to compare the dosimetric characteristics of single and multi-channel cylinders and utilize dose point optimization in a volume model to investigate the feasibility, and the optimum loading method for a multi-ring inflatable intravaginal applicator.

\section{Material and methods}

An inflatable applicator (Capri ${ }^{\circledR}$, Varian, Palo Alto, CA, USA; Fig. 1) with thirteen lumina is designed to reduce air gaps, and conformed dose to patient tissues. The lumina are arranged in two concentric rings (with six lumina each) surrounding a central lumen. The physical dimensions

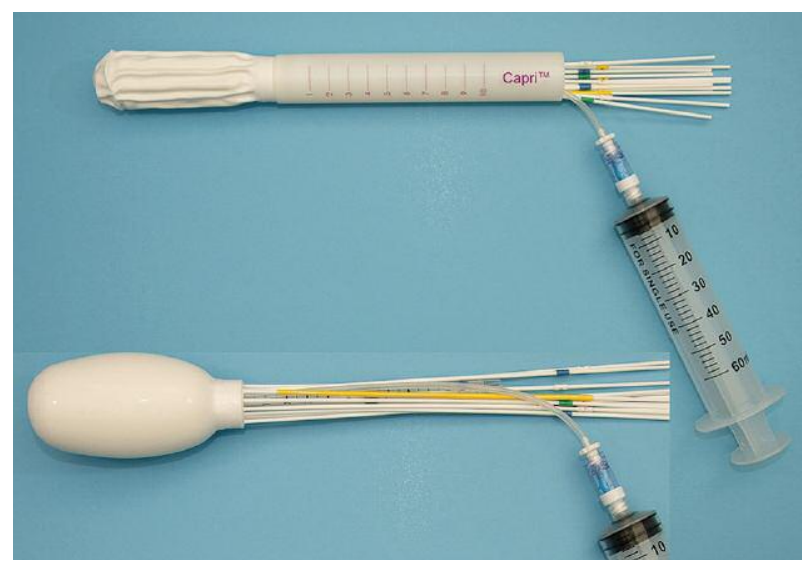

Fig. 1. Capri ${ }^{\circledR}$ with handle accessory in proper position (top) and body of the applicator inflated via the fill port (bottom) of the Capri ${ }^{\circledR}$ applicator with and without infused water are listed in Table 1 . This study investigates the feasibility of the multi-ring design of the Capri ${ }^{\circledR}$ applicator and the intra-vaginal dose distribution between the Capri ${ }^{\circledR}$ applicator, multi-channel cylinder, Contura ${ }^{\circledR}$ balloon, and Mammosite ${ }^{\circledR}$ balloon (Fig. 2A). Computed tomography (CT) images were scanned with $1 \mathrm{~mm}$ slice thickness to have better image resolution for reconstruction of the lumina and delineation of the balloon and its expansion. Plans were generated using Oncentra TPS Version $1.3^{\circledR}$ (Nucletron, an Elekta company, Elekta AB, Stockholm, Sweden). Dose point optimization in a volume model was applied on the surface of the target volume and followed by manually adjusting isodose lines (graphic optimization) to achieve the plan criteria. In dose point optimization, each point dose is calculated based on TG-43 with anisotropic correction; the dwell time to each source position is the analytical solution solved with singular value decomposition (SVD) such that each point receives the reference dose. The plan criteria are to achieve a similar $\mathrm{D}_{90}$ (dose to $90 \%$ of the target volume, about $91 \%$ in this study) and to minimize $\mathrm{V}_{150}$ (fraction of target volume received $150 \%$ or more of the prescribed dose). Source dwell positions were arranged in $2.5 \mathrm{~mm}$ step size, and dose was calculated with a $1 \mathrm{~mm}$ grid size.

\section{Feasibility of the multi-ring applicator}

To study the dosimetric characteristics of a multi-ring cylinder, a PDD (percent depth dose profile) of Ir-192 generated from the central lumen of the cylinder was used as reference to compare the dose at $1 \mathrm{~mm}, 2 \mathrm{~mm}, 3 \mathrm{~mm}, 4 \mathrm{~mm}$ and $5 \mathrm{~mm}$ away from the surface of the cylinder, and $1 \mathrm{~mm}$, $2 \mathrm{~mm}$ and $3 \mathrm{~mm}$ away from the surface of dose prescription. The volume doses of a $1 \mathrm{~mm}$ thickness shell for the respective extension from the surface of the cylinder were collected. To test the feasibility of 6 lumina in the outer ring and 6 lumina in the middle ring, another 6 lumina were drawn and placed between the original outer 6 lumina resulting in 12 equally spaced lumina in the outer ring. Plans were then generated for (cf. Fig. 2C): (1) central single lumen loaded only (central loading - CL); (2) 6 lumina in the outer ring loaded only (peripheral loading - PL); (3) 6 lu-

Table 1. Physical dimension of Capri ${ }^{\circledR}$ (when different amount of water was infused), multi-channel cylinder and Contura ${ }^{\circledR}$

\begin{tabular}{lccc} 
Infused water & Maximum diameter & Dos $^{*}$ & Dmc $^{\star *}$ \\
\hline $0 \mathrm{cc}$ & $28 \mathrm{~mm}$ & $4.5 \mathrm{~mm}$ & $6.5 \mathrm{~mm}$ \\
\hline $20 \mathrm{cc}$ & $33 \mathrm{~mm}$ & $4.8 \mathrm{~mm}$ & $7.5 \mathrm{~mm}$ \\
\hline $30 \mathrm{cc}$ & $35 \mathrm{~mm}$ & $5 \mathrm{~mm}$ & $7.5 \mathrm{~mm}$ \\
\hline $40 \mathrm{cc}$ & $37.5 \mathrm{~mm}$ & $5 \mathrm{~mm}$ & $7.5 \mathrm{~mm}$ \\
\hline $50 \mathrm{cc}$ & $40 \mathrm{~mm}$ & $5 \mathrm{~mm}$ & $7.8 \mathrm{~mm}$ \\
\hline $60 \mathrm{cc}$ & $42 \mathrm{~mm}$ & $5 \mathrm{~mm}$ & $8 \mathrm{~mm}$ \\
\hline Multi-channel & $35 \mathrm{~mm}$ & $4.5 \mathrm{~mm}$ & $\times$ \\
\hline Contura & $35 \mathrm{~mm}$ & $\times$ & $5 \mathrm{~mm}$
\end{tabular}

Total physical length of Capri® is $9 \mathrm{~cm}$.

${ }^{*}$ Dos: Distance from outer ring to the surface of Capri® applicator.

${ }^{*}$ Dmc: Distance from middle ring to center lumen of Capri® applicator. 


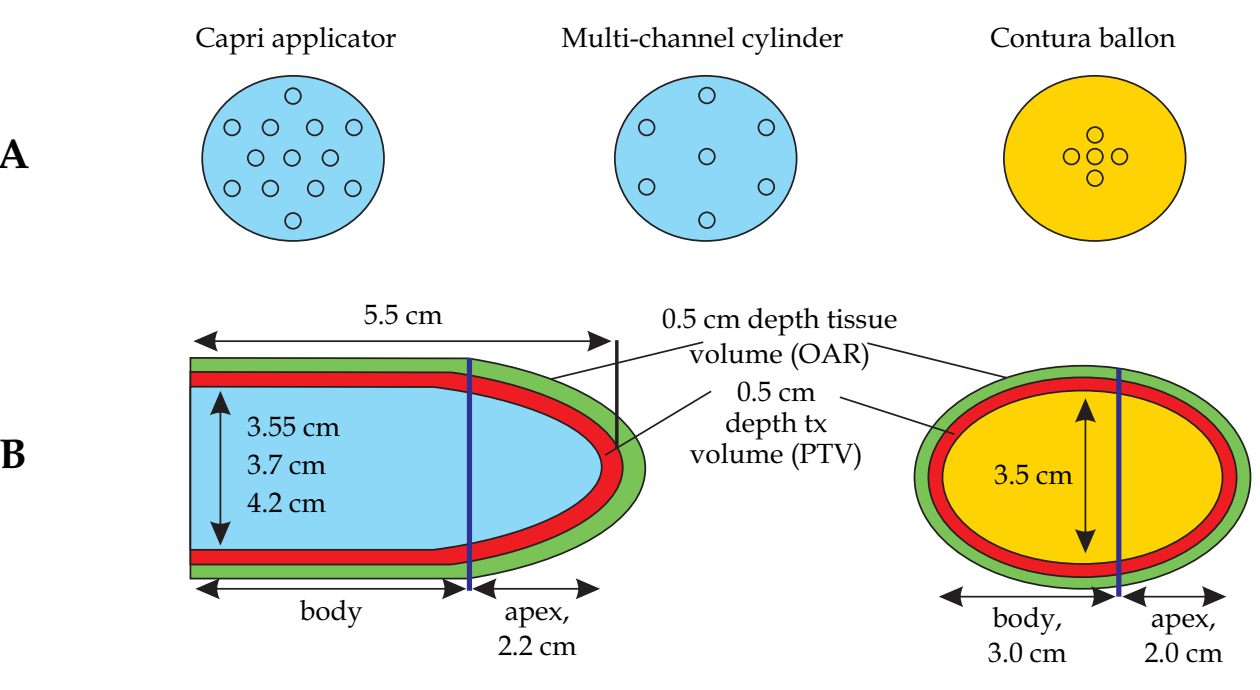

A
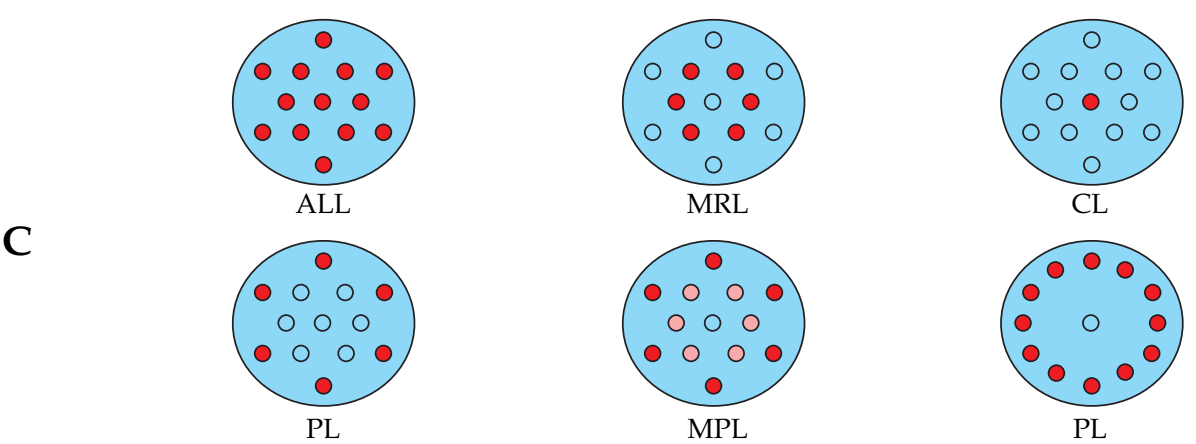

Fig. 2. A) Lumen configuration of different multi-channel applicators; B) PTV volume and OAR volume at portions of body and apex for cylinder type (left) and Mammosite ${ }^{\circledR}$ type (right) applicators; C) configuration of different loading techniques used in this study. The diameters of the Capri ${ }^{\circledR}$ applicator in (B) correspond to $30 \mathrm{cc}, 40 \mathrm{cc}$, and $60 \mathrm{cc}$ infused water (see Table 1)

mina in the outer ring loaded combined with $30 \%$ loading in the middle ring 6 lumina (modified peripheral loading - MPL); (4) 12 lumina in the outer ring loaded only (peripheral loading with 12 lumina - PL12).

\section{Dosimetric comparison between different multi-channel applicators and loading patterns}

Plans were generated from three multi-channel applicators: a $3.5 \mathrm{~cm}$ diameter regular multi-channel cylinder, a multi-ring Capri ${ }^{\circledR}$ balloon, and a Contura ${ }^{\circledR}$ balloon. Capri $^{\circledR}$ of different sizes were planned with CL, PL, MPL, middle ring lumina loaded only (MRL), and all lumina loaded (ALL). The multi-channel cylinder was planned with CL and PL (Fig. 2B). The plan with the Mammosite ${ }^{\circledR}$ was simulated using the Contura ${ }^{\circledR}$ applicator with central loading only. Dose was prescribed to $5 \mathrm{~mm}$ depth (PTV) from the surface of each applicator $\left(\mathrm{D}_{90}>90 \%\right)$ with minimum dose to the normal tissue (organs at risk - OAR), which was another $5 \mathrm{~mm}$ away (cf. Fig. 2B). To study the difference of the dose to the apex of the vagina and the dose to the body of vagina, plans were evaluated at the proximal $2 \mathrm{~cm}$ of the vagina [PTV_A $\left(\mathrm{V}_{150}\right)$ \& OAR_A $\left.\left(\mathrm{V}_{80}\right)\right]$ $\left(\mathrm{V}_{80}, \mathrm{OAR}\right.$ volume fraction received $80 \%$ of prescribed dose) and at the rest of the treatment length of the vagina body $\left[P T V \_B\left(V_{150}\right)\right.$ \& OAR_B $\left.\left(V_{80}\right)\right]$.

\section{Non-uniform prescription depth around the multi-ring cylinder}

To assess the advantage of the inflatable multi-ring applicator, four inserts in two different patients were scanned with CT and the images were exported to the TPS for study. The PTV was defined as a $5 \mathrm{~mm}$ expansion of the volume of the inflated balloon from which the overlapping volumes of the bladder and rectum were subtracted. Plans were compared for CL, PL and peripheral loading without loading the anterior and posterior lumina (lateral loading - LL), MPL, and modified peripheral loading without loading the anterior and posterior lumina (MLL). With similar $D_{90}$ to the PTV, plans were evaluated at $\mathrm{D}_{95}$ and $\mathrm{V}_{150}$ of the PTV, $0.1 \mathrm{cc}, 2 \mathrm{cc}$ and $5 \mathrm{cc}$ of bladder and rectum, and a conformal index (COIN) was calculated which is defined as [15]

$$
\text { COIN }=\frac{P T V_{95 \%}}{P T V} \times \frac{P T V_{95 \%}^{\prime}}{T V_{95 \%}}
$$

where $P T V_{95 \%} / P T V$ is the fraction of planning volume (without volume of balloon) receiving $95 \%$ of the prescribed dose, $P T V_{95 \%}^{\prime}$ and $T V_{95 \%}$ are the planning volume (including balloon) and the entire treatment volume receiving $95 \%$ of the prescribed dose, respectively. 

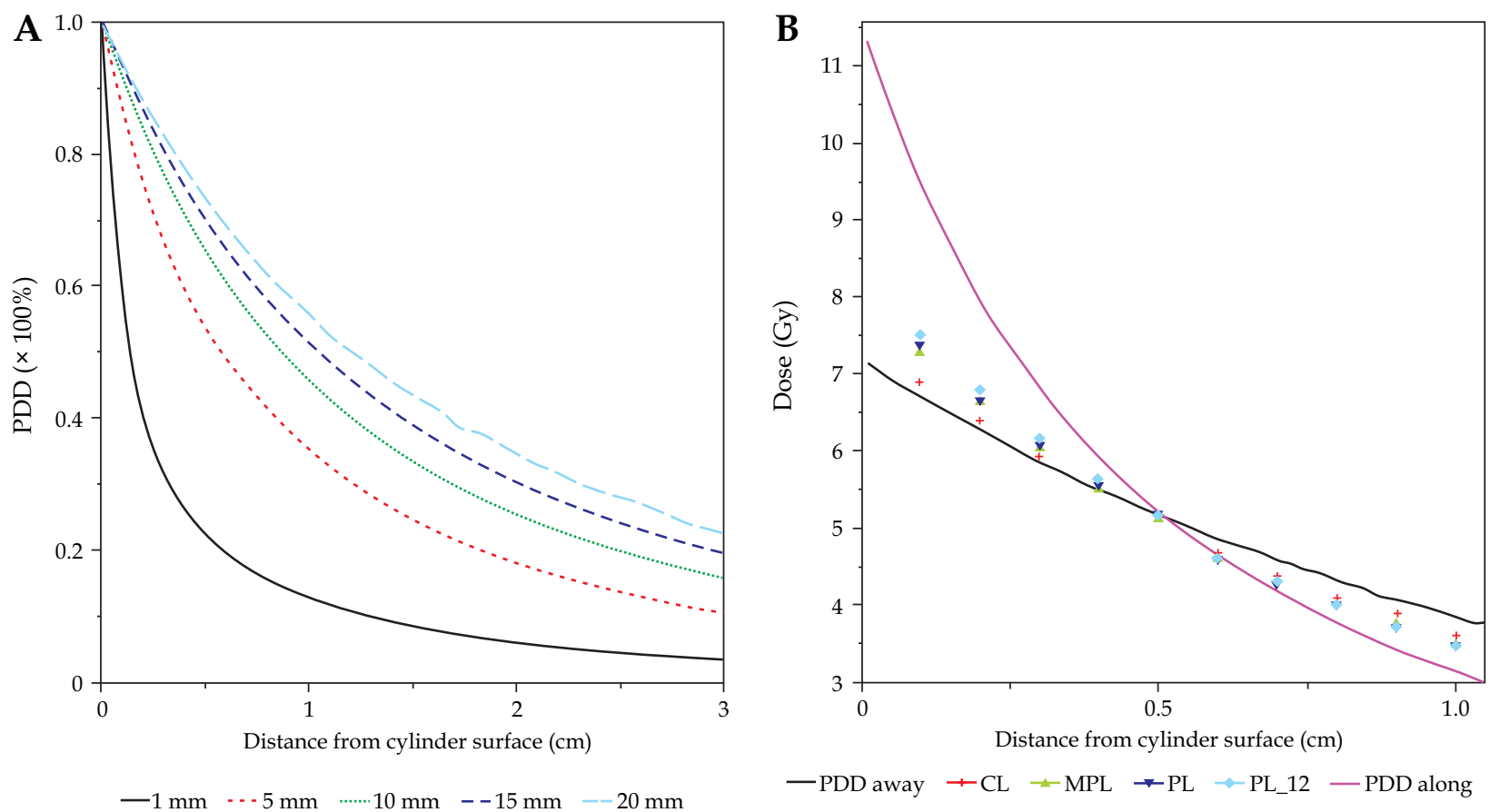

Fig. 3.A) The percent depth dose profile (PDD) from cylinder surface with different radius; B) the dose profile parallel (along) and perpendicular (away) to the central loading cylinder (of $3.5 \mathrm{~cm}$ in diameter) and the mean volume dose at different depths for various loading techniques

\section{Results}

\section{Feasibility of multi-ring applicator}

Figure 3A shows the PDDs of Ir-192 from a $5 \mathrm{~cm}$ active length of cylinder for a number of different radii. Each curve was normalized to the surface of the cylinder and was taken from the lateral cut of the cylinder. It is the inverse square law from the point source which allows the use of brachytherapy sources to deposit high dose to the implanted lesion and spare dose to normal tissue. The steepness of the dose gradient as measured form the surface depends on the

Table 2. Dose (Gy) statistic (mean \pm 2 SD) from inner shell volume (starts from $V_{1} \mathrm{~mm}$ ) to outer shell volume (end at $V_{10} \mathrm{~mm}$ ) for different loading technique

\begin{tabular}{ccccc} 
& $\mathrm{CL}$ & $\mathrm{MPL}$ & $\mathrm{PL}$ & $\mathrm{PL} 12$ \\
\hline $\mathrm{V}_{1 \mathrm{~mm}}$ & $6.88 \pm 1.05$ & $7.28 \pm 0.99$ & $7.37 \pm 1.02$ & $7.52 \pm 1.48$ \\
\hline $\mathrm{V}_{2 \mathrm{~mm}}$ & $6.38 \pm 0.88$ & $6.62 \pm 0.79$ & $6.65 \pm 0.75$ & $6.79 \pm 1.08$ \\
\hline $\mathrm{V}_{3 \mathrm{~mm}}$ & $5.91 \pm 0.76$ & $6.04 \pm 0.66$ & $6.07 \pm 0.62$ & $6.16 \pm 0.83$ \\
\hline $\mathrm{V}_{4 \mathrm{~mm}}$ & $5.51 \pm 0.68$ & $5.55 \pm 0.61$ & $5.56 \pm 0.59$ & $5.65 \pm 0.68$ \\
\hline$V_{5 \mathrm{~mm}}$ & $5.15 \pm 0.65$ & $5.13 \pm 0.57$ & $5.14 \pm 0.55$ & $5.19 \pm 0.58$ \\
\hline$V_{6 \mathrm{~mm}}$ & $4.66 \pm 0.60$ & $4.60 \pm 0.52$ & $4.58 \pm 0.52$ & $4.62 \pm 0.33$ \\
\hline$V_{7 \mathrm{~mm}}$ & $4.37 \pm 0.59$ & $4.29 \pm 0.51$ & $4.25 \pm 0.50$ & $4.31 \pm 0.46$ \\
\hline$V_{8 \mathrm{~mm}}$ & $4.08 \pm 0.60$ & $4.00 \pm 0.50$ & $4.00 \pm 0.50$ & $4.01 \pm 0.47$ \\
\hline$V_{9 \mathrm{~mm}}$ & $3.86 \pm 0.60$ & $3.73 \pm 0.49$ & $3.71 \pm 0.49$ & $3.73 \pm 0.46$ \\
\hline$V_{10 \mathrm{~mm}}$ & $3.59 \pm 0.60$ & $3.47 \pm 0.47$ & $3.46 \pm 0.49$ & $3.47 \pm 0.44$
\end{tabular}

$C L$ - central loading, MPL - modified peripheral loading, $P L$ - peripheral loading, PL12 - peripheral loading with 12 lumina radius of the cylinder. The smaller the radius of the cylinder, the sharper the PDD is. The solid curves in Fig. 3B represent the dose range outside the surface of a $35 \mathrm{~mm}$ diameter cylinder. The upper solid curve represents the dose profile perpendicular to the cylinder; while the lower solid curve represents the dose profile along the tip of cylinder. The discrete symbols illustrate the average dose received by a volume shell at different distances from the cylinder surface. The mean and range of dose in the corresponding volumes are summarized in Table 2. As can be seen from Table 2, CL has fewer hot spots within the PTV (5 mm), but has higher dose outside the PTV. All of the peripheral loading techniques yielded similar low dose outside the PTV, however, PL12 had the highest hot spot within the PTV and MPL had the lowest hot spot among these three techniques.

\section{Dosimetric comparison between different multi-channel applicators}

The three bar graphs shown on the bottom of Fig. 4 compare cylinders having a diameter of $35 \mathrm{~mm}$ for the different applicators. As can be seen from Fig. 4 for the body section of the cylinder, both Contura ${ }^{\circledR}$ and Mammosite ${ }^{\circledR}$ had the lowest OAR_B $\left(\mathrm{V}_{80}\right)$, but the highest PTV_B $\left(\mathrm{V}_{150}\right)$, while Capri ${ }^{\circledR}$ planned with PL or MPL had slightly better OAR_B $\left(\mathrm{V}_{80}\right)$ and reasonable PTV_B $\left(\mathrm{V}_{150}\right)$ compared with the multi-channel cylinder. At the apical portion of the cylinder, both Contura ${ }^{\circledR}$ and Mammosite ${ }^{\circledR}$ had similar PTV_A $\left(\mathrm{V}_{150}\right)$ and better OAR_A $\left(\mathrm{V}_{80}\right)$ compared with Capri ${ }^{\circledR}$. The multi-channel cylinder had the highest value in both PTV_A( $\left.\mathrm{V}_{150}\right)$ and OAR_A $\left(\mathrm{V}_{80}\right)$. The upper 12 bar graphs shown in Fig. 4 compare different configurations of activated lumina in the Capri ${ }^{\circledR}$ cylinder for different di- 


\section{A}

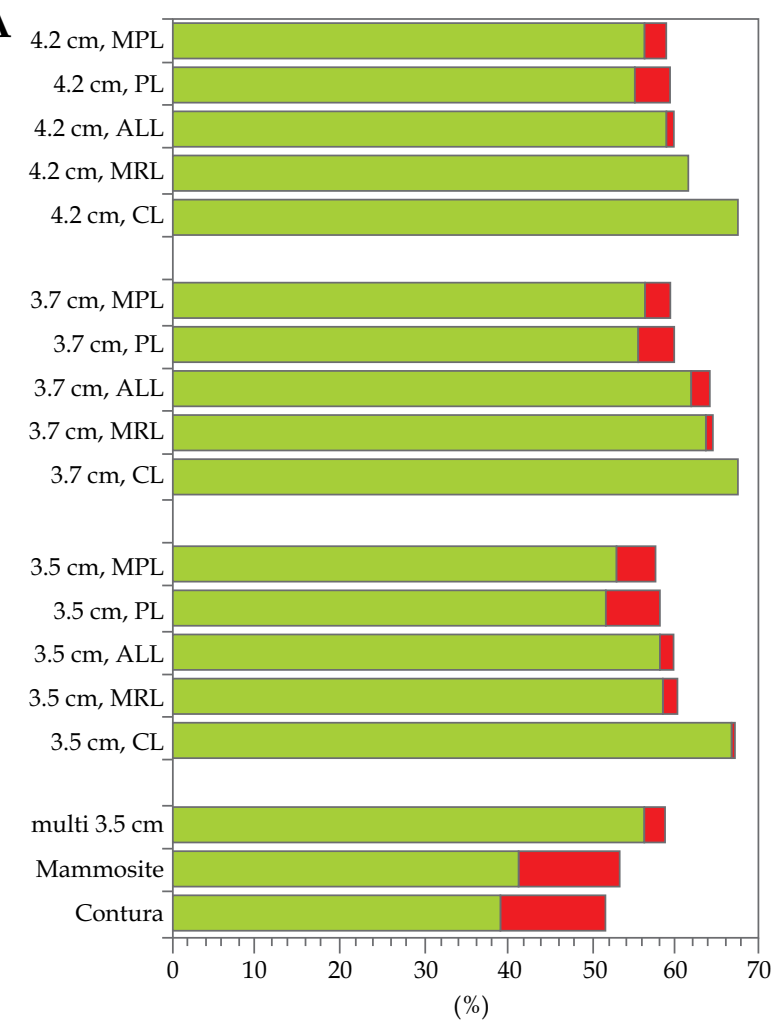

$\square$ PTV_B $\left(\mathrm{V}_{150}\right) \quad \square$ OAR_B $\left(\mathrm{V}_{80}\right)$
B

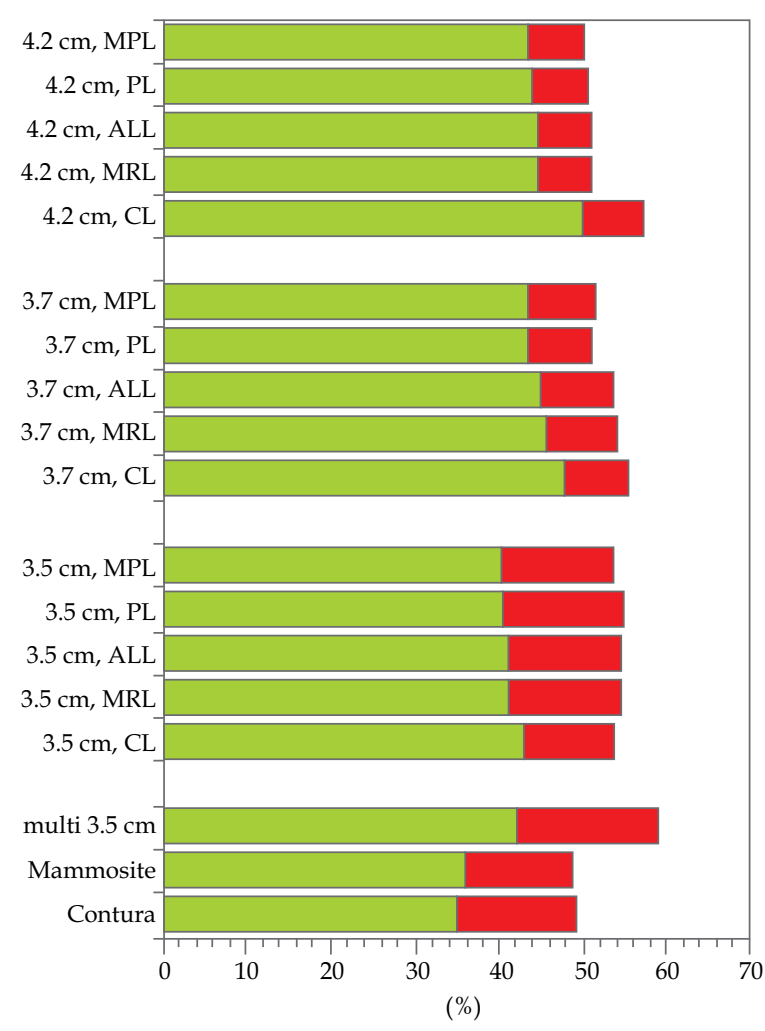

$\square$ PTV_A $\left(\mathrm{V}_{150}\right) \quad \square$ OAR_A $\left(\mathrm{V}_{80}\right)$

Fig. 4. Percent volume of OAR and PTV receiving $80 \%$ and $150 \%$ of the prescribed dose, respectively, in body (A) and apex (B). Device and loading patterns are depicted in Figure 1

ameters. Plans with CL or MRL showed better PTV $\left(\mathrm{V}_{150}\right)$, but worse $\mathrm{OAR}\left(\mathrm{V}_{80}\right)$. On the other hand, plans with PL showed better $\operatorname{OAR}\left(\mathrm{V}_{80}\right)$, but worse $\operatorname{PTV}\left(\mathrm{V}_{150}\right)$. Plans with ALL had PTV $\left(V_{150}\right)$ close to plans with CL \& MRL, but the $\operatorname{OAR}\left(\mathrm{V}_{80}\right)$ was not optimal. To minimize PTV $\left(\mathrm{V}_{150}\right)$ and OAR $\left(\mathrm{V}_{80}\right)$ simultaneously, plans with MPL were the best choice for all sizes. They offered the least amount of $\mathrm{OAR}\left(\mathrm{V}_{80}\right)$ which were close to plans to the $\mathrm{CL}$ and maintained moderate values of PTV(150).

\section{Non-uniform prescription depth around the multi-ring cylinder}

Table 3 shows the results for different loading techniques in clinical patients. Both CL and LL had low conformality index values. Central loading could not conform the dose shape to the target and had a higher dose to the volume of bladder and rectum adjacent to the PTV. Lateral loading resulted in bulged out high dose (cf. Fig. 5C and 5D) toward two sides and delivered $150 \%$ of prescription dose to more than $25 \%$ of the PTV. The PL and MLL gave similar conformal dose distributions, low dose to the OAR, and slightly higher $\mathrm{V}_{150}$ in the PTV. Modified peripheral loading (cf. Fig. 5A and 5D) had the best conformality index, delivered low dose to the OAR, and had similar low PTV $\left(\mathrm{V}_{150}\right)$ compared to central loading.

\section{Discussion}

Dose profiles from an Ir-192 source are the basis of dose distributions produced from any applicator design used in HDR brachytherapy, especially for the intra-vaginal applicators discussed. The dose gradient on the surface of a $1 \mathrm{~mm}$ radius cylinder (e.g., tandem in clinical case) had

Table 3. Summary (mean \pm 2 SD) of planning results from different loading techniques for a non-homogeneous prescription depth around multi-ring cylinder

\begin{tabular}{lccccccc} 
& $\mathrm{D}_{95 P T V}$ & $\mathrm{~V}_{150 \mathrm{PTV}}$ & $\mathrm{COIN}$ & $\mathrm{D}_{0.1 \mathrm{cc}}(\mathrm{B})$ & $\mathrm{D}_{2 \mathrm{cc}}(\mathrm{B})$ & $\mathrm{D}_{0.1 \mathrm{cc}}(\mathrm{R})$ & $\mathrm{D}_{2 \mathrm{cc}}(\mathrm{R})$ \\
\hline $\mathrm{MPL}$ & $95.2 \pm 0.0$ & $12.9 \pm 2.5$ & $79.0 \pm 3.5$ & $4.8 \pm 0.3$ & $3.8 \pm 0.3$ & $5.6 \pm 0.4$ & $4.4 \pm 0.1$ \\
\hline $\mathrm{MLL}$ & $95.2 \pm 0.1$ & $16.3 \pm 2.2$ & $75.1 \pm 4.5$ & $4.9 \pm 0.3$ & $3.9 \pm 0.3$ & $5.8 \pm 0.4$ & $4.5 \pm 0.1$ \\
\hline $\mathrm{PL}$ & $95.2 \pm 0.0$ & $17.0 \pm 1.4$ & $74.6 \pm 5.7$ & $5.0 \pm 0.5$ & $3.9 \pm 0.5$ & $5.5 \pm 0.2$ & $4.5 \pm 0.3$ \\
\hline $\mathrm{LL}$ & $95.2 \pm 0.1$ & $28.7 \pm 4.5$ & $69.2 \pm 3.5$ & $5.0 \pm 0.6$ & $3.9 \pm 0.5$ & $5.6 \pm 0.4$ & $4.5 \pm 0.1$ \\
\hline $\mathrm{CL}$ & $95.2 \pm 0.1$ & $12.6 \pm 1.4$ & $71.6 \pm 3.9$ & $5.1 \pm 0.6$ & $4.0 \pm 0.4$ & $6.3 \pm 0.3$ & $5.0 \pm 0.3$
\end{tabular}

Unit: $D_{95}, \%$ of prescribed dose; $V_{150}, c c ; D_{0.1 c c}$ and $D_{2 c c} G y$. 

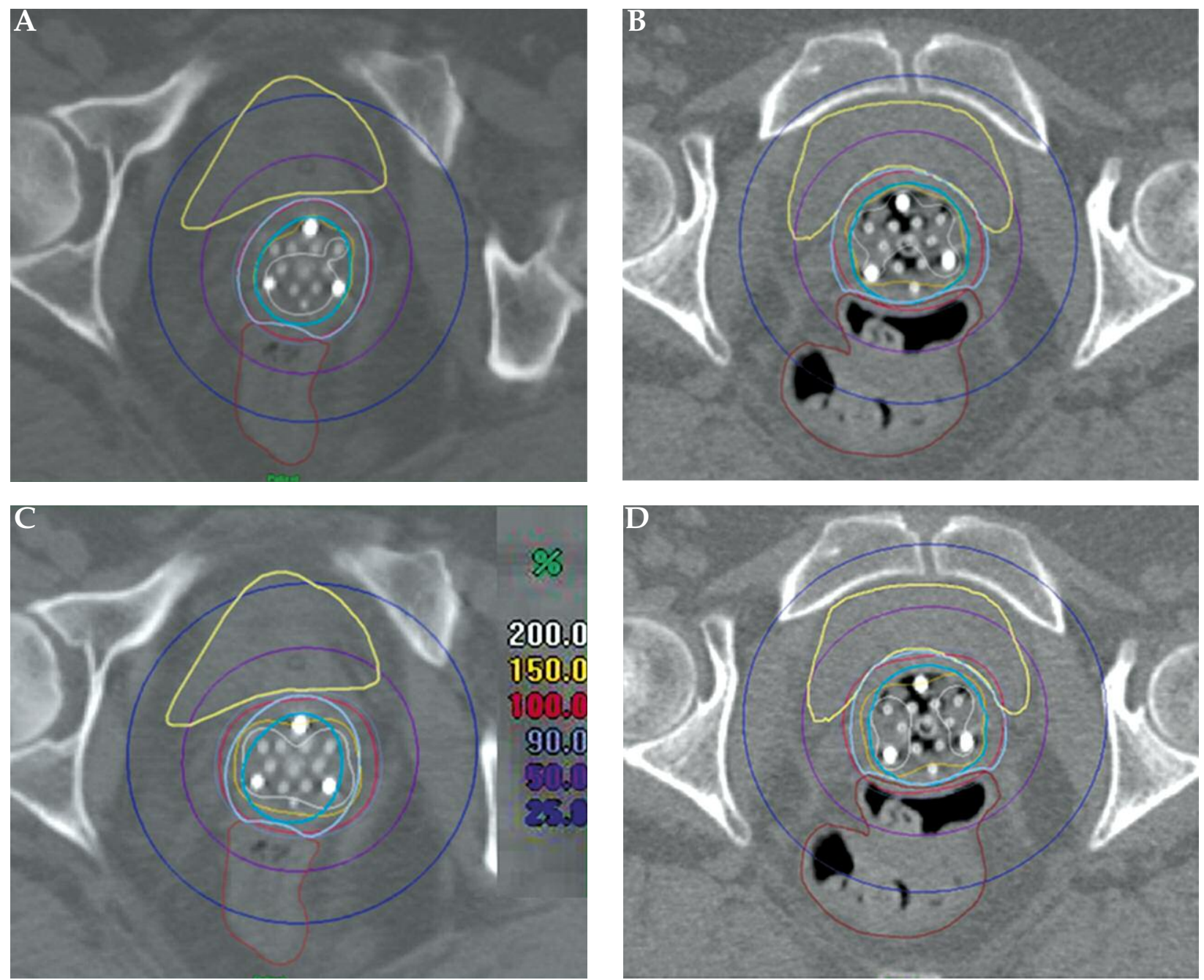

Fig. 5. Dose distributions of modified peripheral loading (A, B) and lateral loading with anterior/posterior lumina unloaded to spare bladder and rectum (C, D). Yellow: bladder; brown: rectum; cyan: balloon; light blue: PTV

a $70 \%$ drop in the first $3 \mathrm{~mm}$ from the surface of the cylinder and an $80 \%$ drop in the first $5 \mathrm{~mm}$ from the surface of the cylinder. There was a $20 \%$ and $25 \%$ decrease in the first $3 \mathrm{~mm}$ and $5 \mathrm{~mm}$, respectively, for the $15 \mathrm{~mm}$ radius cylinder. A typical prescription depth of $5 \mathrm{~mm}$ resulted in a $33 \%$ dose increase at the surface for a $15 \mathrm{~mm}$ radius cylinder. The surface dose was 5 times the prescription dose for a $1 \mathrm{~mm}$ radius cylinder. For cylinders with a radius of less than $10 \mathrm{~mm}$ the dose fall off from $5 \mathrm{~mm}$ to $10 \mathrm{~mm}$ from the surface was less steep than from $0 \mathrm{~mm}$ to $5 \mathrm{~mm}$. For cylinders having a radius equal or larger than $10 \mathrm{~mm}$ the change in dose gradient beyond $5 \mathrm{~mm}$ from the surface of the cylinder was small. This means that the dose sparing to normal tissue beyond $5 \mathrm{~mm}$ prescription depth is similar for mid to large sized cylinders. Clinically, in addition to providing better immobilization, use of the maximum size cylinder during intra-vaginal implant can decrease the surface dose to the inner vaginal mucosa.

The radius at the apical portion of a cylinder (typically $7-8 \mathrm{~mm}$ from the first dwell position) is smaller than the radius at the cylinder body. This, combined with the anisotropic source configuration within the cylinder as well as the source shape itself, results in the dose profile parallel to the cylinder axis (the curve of 'PDD along' in Fig. 3B) having a higher gradient from cylinder tip than from the cylinder body (the curve of 'PDD away' in Fig. 3B). The example shown in Fig. 3B demonstrates that surface dose ranged from $40 \%$ to $120 \%$ (from body portion to apex portion) relative to the dose $5 \mathrm{~mm}$ from the surface of a $35 \mathrm{~mm}$ diameter cylinder with central loading. Mean volume dose at shallow depths for the multi-channel design was noticeably higher than for central loading. However, the benefit of reducing dose beyond the prescription depth was low. Surprisingly, applying peripheral loading with 12 lumina (PL12) did not reduce dose to normal tissue as compared to peripheral loading using 6 lumina (PL or MPL), but instead increased hot spots to the vaginal mucosa. Consider three neighboring dwell positions in the same axial plane, any point on the front of the cylinder surface has a shorter total distance (point to dwell positions) from the 12 lumina peripheral configuration than from the 6 lumina peripheral configuration. At points close to the cylinder surface, dwell positions which are close to the points have a high relative dose contribution. However, at points which are far- 
ther away from the cylinder surface, dose contributed from the front end dwell positions does not differ dramatically from the dose contributed from the opposite end. Unless the prescription depth is on the surface or very shallow, the benefit of a 12 lumina peripheral loaded cylinder is very limited. If one considers a uniformly prescribed depth only, the dose distribution beyond the surface of a cylinder is mainly determined by the distance from the source. The radius at the apical end of a cylinder applicator gradually decreases. In this study we have split the cylinder into an apical section and a body section. In this way, the dosimetric impact of different multi-channel cylinders and different loading techniques on these two sections can be evaluated separately and the results will be less sensitive to the total treatment length $(\mathrm{cm})$.

Contura ${ }^{\circledR}$ and Mammosite ${ }^{\circledR}$ applicators produced elliptical dose shapes and had mean diameters less than their maximum diameters. Implanting Mammosite ${ }^{\circledR}$ (or Contu$\mathrm{ra}^{\circledR}$ ) balloons to treat the vaginal cuff shows excellent dose sparing in normal tissue. However, the dose to the vaginal mucosa is high. This may increase the risk of vaginal stenosis, especially when total dose is high or a high dose rate is applied [16]. Cylindrical applicators gave a higher dose to normal tissue in both the apical and body sections. For the multi-channel cylinder, the peripheral lumina reduced the dose to normal tissue compared to central loading alone at the expense of a slight dose increase to the surface of the vaginal mucosa. Similar to results of the studies above, MPL helped to reduce the mucosa dose. As to the dose in different sections of the cylinder, the apical section had similar dose results to what could be achieved by using a Mammosite ${ }^{\circledR}$ balloon, which gives higher dose to mucosa and less dose to normal tissue. However, dose to the body of the cylinder was reversed and gave higher dose to normal tissue, but less dose to the mucosa. The design of the Capri ${ }^{\circledR}$ applicator does not have an outer ring of lumina extending into the apical section, there are only lumina in the middle and central lumen in this section. This results in a reduction in mucosa dose compared to other solid type multi-channel cylinders.

Different loading techniques applied in the Capri ${ }^{\circledR}$ applicator for a uniform prescription depth resulted in slightly different dose distributions. Normal tissue received lower doses if the dose to vaginal mucosa was primarily deposited due to dwell positions in the outer ring. In terms of normal tissue sparing, we found that CL was the worst, MRL \& ALL were better and PL \& MPL were the best. A loading combination in the outer and central ring has been tested and the dosimetric results were similar to MRL. These results apply to all cylinder diameters explored. A larger diameter slightly increased the dose to normal tissue with the same loading technique. Larger cylinders also reduced high dose to the mucosa especially in the apical section.

Since $95 \%$ of vaginal lymphatic channels are located within $3 \mathrm{~mm}$ from the vaginal surface [17], for postoperative endometrial brachytherapy, a target volume consisting of a $5 \mathrm{~mm}$ expansion from the surface of the cylinder (excluding any overlap of the bladder and rectum) should be adequate to cover the vaginal lymphatic channels located within the vaginal wall. A multi-channel cylinder can be beneficial to non-uniform treatment depths around a cylinder. Demanes et al. [10] reported their experience of using CET vaginal applicators, which have a $26 \mathrm{~mm}$ outer diameter and a $2 \mathrm{~mm}$ space between the peripheral source positions and the vaginal mucosa. By not loading the anterior and posterior lumina (lateral loading, the LL pattern in this study), the mean bladder (point on X-ray film) dose decreased from $87 \%$ to $73 \%$ and the mean rectum (point) dose decreased from $91 \%$ to $75.9 \%$ compared to central loading only. However, in a three-dimensional volume plan with a CT image, LL does not necessarily spare dose to OAR as compared to peripheral loading if the same target volume coverage is considered. In the case of our study, each CT image showed that the inflatable balloon opened the folds and wrinkles of the intravaginal cavity and adapted to the vaginal wall very well. Changing from CL to LL loading patterns, the dose to $0.1 \mathrm{cc}$ of rectum decreased by $14 \%$ of the prescription dose. Dose to the bladder followed a similar trend. In terms of COIN, LL was as low as CL. Central loading and LL loading patterns differed in that CL over-treated OAR adjacent to the target, whereas LL over-treated the vaginal mucosa and produced bulging dose distributions into the muscular layer of the vaginal wall.

Bladder and rectum, as well as the intestines, are QAR and need to be spared in intravaginal brachytherapy. In the study by Jereczek et al. [18], the acute effects to the bowel, bladder, and vaginal wall were $66 \%, 36 \%$, and $11 \%$, respectively. The observed late effects were $41 \%, 21 \%$ and $15 \%$, respectively. Onsrund et al. [19] reported that when using a standardized $5 \mathrm{~mm}$ prescription depth, the late effects to the bladder, vaginal wall, and rectum were $11 \%, 34 \%$ and $5 \%$, respectively. The effects reduced to $1 \%, 17 \%$ and $1 \%$, respectively, when an individualized prescription depth was used. Different types of multi-channel cylinders have been developed and use peripheral loading to minimize dose to these organs. It should be noted that compared to central loading, peripheral loading will increase dose to the surface of the vaginal mucosa. Lancaster summarized the literature between 1971 and 2001 and found $24-88 \%$ of patients had vaginal stenosis after treatment with brachytherapy [20]. Recently Bahng et al. [21] reported 33\% had grade 1 and $14 \%$ had grade 2 or 3 vaginal toxicity in endometrial HDR brachytherapy. They found strong correlations between age, total dose, dose rate, and treatment length and the incidence of vaginal complications. ESTRO recommends having the $150 \%$ isodose confined within the surface of the cylinder [4].

In this study, we compared different applicators for intravaginal brachytherapy and different loading methods. We first demonstrated the high dose gradient nature of brachytherapy source when source is close to the point of interest. The dosimetry of multi-channel cylinder is constrained by the physics of dose deposition with an Ir-192 source together with the symmetric nature of the applicator. A better optimization may improve slightly the dose distribution with the use of multi-channel cylinder, however, it shouldn't change the conclusion from our finding. We found modified peripheral loading with a multi-ring cylinder had the advantage of allowing the optimization of dose to the target (vaginal mucosa) and the OAR (bladder and rectum). If a concentric dose distribution with equal depth 
around the cylinder is needed, limiting the mucosal dose, it is possible that only one central catheter may be the optimum solution when medium to large cylinder size is applied. If an asymmetric dose distribution is needed, in order to treat a non-homogeneous target volume or to spare a nearby OAR, the addition of non-central catheters shows promising results. On the other hand, activating too many lumina peripherally does not show further advantages in reducing dose to OAR. The lateral loading technique should be carefully applied in order to prevent high dose to the mucosa and the muscular layer of the vaginal wall.

\section{Conclusions}

In addition to the potential advantage of reducing air gaps between the applicator and the vaginal mucosa, an inflatable design would make the implant more comfortable for the patients and could maximize the applicator diameter to reduce hot spots in the vaginal mucosa. In the treatment of a non-homogeneous vaginal wall, multi-channel cylinders allow for individualized optimal dose distributions that can better conform to the target. An additional middle ring of lumina offers flexibility in treatment patterns to balance target coverage, dose uniformity in the vaginal mucosa, and dose sparing to the rectum and bladder. This study provides a preliminary evaluation of the feasibility of the Capri ${ }^{\circledR}$ applicator in treating the vaginal mucosa. Further clinical studies of the Capri ${ }^{\circledR}$ applicator in terms of fixation, patient comfort during application, and acute toxicity are warranted.

\section{Acknowledgements}

Part of the data has been presented in the combined conference of ESTRO and ABS 2012 in Barcelona, Spain.

\section{References}

1. Nag S, Erickson B, Parikh S et al. The American Brachytherapy Society recommendations for high-dose-rate brachytherapy carcinoma of the endometrium. Int J Radiat Oncol Biol Phys 2000; 48: 779-790.

2. Lin LL, Grigsby PW, Powell MA et al. Definitive radiotherapy in the management of isolated vaginal recurrences of endometrial cancer. Int J Radiat Oncol Biol Phys 2005; 63: 500-504.

3. Jhingran A, Burke TW, Eifel PJ. Definitive radiotherapy for patients with isolated vaginal recurrence of endometrial carcinoma after hysterectomy. Int J Radiat Oncol Biol Phys 2003; 56: 1366-1372.

4. Pötter R, Gerbaulet A, Haie-Meder C. Endometrial cancer. In Gerbaulet A, Potter R, Mazeron JJ, et al. (eds.). The GEC ESTRO Handbook of Brachytherapy. ESTRO 2002, pp. 365-401.

5. Small W, Erickson B, Kwakwa F. American Brachytherapy Society survey regarding practice patterns of postoperative irradiation for endometrial cancer: current status of vaginal brachytherapy. Int J Radiat Oncol Biol Phys 2005; 63: 1502-1507.

6. Cameron AL, Cornes P, Al-Booz H. Brachytherapy in endometrial cancer: quantification of air gaps around a vaginal cylinder. Brachytherapy 2008; 7: 355-358.

7. Richardson S, Palaniswaamy G, Grigsby PW. Dosimetric effects of air pockets around High-Dose Rate brachytherapy vaginal cylinders. Int J Radiat Oncol Biol Phys 2010; 78: 276-279.

8. Miller DA, Richardson S, Grigsby PW. A new method of anatomically conformal vaginal cuff HDR brachytherapy. Gynecol Oncol 2010; 116: 413-418.
9. Sowards KT, El-Ghamry MN. An inter-comparison of differing applicators for HDR vaginal cuff brachytherapy with focus on the potential use of the Contura device. Int J Radiat Oncol Biol Phys 2010; 78: S417.

10. Demanes DJ, Rege $S$, Rodriguez RR et al. The use and advantages of a multichannel vaginal cylinder in High-Dose-Rate brachytherapy. Int J Radiat Oncol Biol Phys 1999; 44: 211-219.

11. Tanderup K, Lindegaard JC. Multi-channel intracavitary vaginal brachytherapy using three-dimensional optimization of source geometry. Radiother Oncol 2004; 70: 81-85.

12. Hansen JW. The importance of applicator design for intraluminal brachytherapy of rectal cancer. Med Phys 2006; 33: 3220-3224.

13. Li S, Aref I, Walker E et al. Effects of prescription depth, cylinder size, treatment length, tip space, and curved end on doses in High-Dose-Rate vaginal brachytherapy. Int J Radiat Oncol Biol Phys 2007; 67: 1268-1277.

14. Sivakumar SS, Solomon JGR, Supe SS et al. Optimization in high dose rate vaginal cylinder for vaginal cuff irradiation. Rep Pract Oncol Radiother 2008; 13: 35-48.

15. Baltas D, Kolotas C, Geramani K et al. A conformal index (COIN) to evaluate implant quality and dose specification in brachytherapy. Int J Radiat Oncol Biol Phys 1998; 40: 512-524.

16. Au SP, Grigsby PW. The irradiation tolerance dose of the proximal vagina. Radiother Oncol 2003; 67: 77-85.

17. Choo JJ, Scudiere J, Bitterman P et al. Vaginal lymphatic channel location and its implication for intravitary brachytherapy radiation treatment. Brachytherapy 2005; 4: 236-240.

18. Jereczek-Fossa BA, Jassem J, Badzio A. Relationship between acute and late normal tissue injury after postoperative radiotherapy in endometrial cancer. Int J Radiat Oncol Biol Phys 2002; 52: 476-482.

19. Onsrund M, Strickert T, Marthinsen ABL. Late reactions after postoperative High-Dose-Rate intravaginal brachytherapy for endometrial cancer: a comparison of standardized and individualized target volumes. Int J Radiat Oncol Biol Phys 2001; 49: 749-755.

20. Lancaster L. Preventing vaginal stenosis after brachytherapy for gynaecological cancer: an overview of Australian practice. European J Oncology Nursing 2004; 8: 30-39.

21. Bahng AY, Dagan A, Bruner DW. Determination of prognostic factors for vaginal mucosal toxicity associated with intravaginal High-Dose Rate brachytherapy in patients with endometrial cancer. Int J Radiat Oncol Biol Phys 2011; 82: 667-673. 